\title{
THE ANDROID APPLICATION FOR SENDING SMS IN REGIONAL
} LANGUAGES

\author{
K. Sreenivasa Ravi ${ }^{1}$, M.Dorathi Mounika ${ }^{2}$, T.Sai Amrutha Varshini ${ }^{3}$, R.Koushik Reddy ${ }^{4}$ \\ ${ }^{1}$ Department of Electronics and Computers, $K$ L University, Andhra Pradesh, India \\ ${ }^{2}$ Department of Electronics and Computers, $K$ L University, Andhra Pradesh, India \\ ${ }^{3}$ Department of Electronics and Computers, $K$ L University, Andhra Pradesh, India \\ ${ }^{4}$ Department of Electronics and Computers, $K$ L University, Andhra Pradesh, India
}

\begin{abstract}
To develop an android based application capable of sending SMS in various languages. Subject to availability of time, number of applications can be developed using this concept. Success in this endeavor will extend the life time of deployed systems, and directly advance our ability to develop systems in new languages and new domains without onerous demands of labeled speech, by essentially creating systems that learn and improve over time. Sending SMS in regional languages application is a product that combines all the useful features of different applications into one single application which mainly concentrates on creating SMS messages in various languages with English phonetics as input and that English phonetics will be converted into selected language by transliteration method. A radio button password is far more secure than a graphical pattern password, the reason being that it doesn't have a pattern to remember. In this project we developed an application that sends SMS in regional languages by transliterating English phonetics to selected language.
\end{abstract}

Keywords: IDE-Eclipse, Eclipse-plug-in-ADT, Software Development Kit, Android Emulator Debugger, Java development kit.

\section{INTRODUCTION}

Mobile Phones have become an Integral part of our everyday Life, causing Higher Demands for content that can be used on them. Smart Phones offer Customer enhanced methods to interact with their phones but the most natural way of interaction remains Speech. Market for Smart mobile phones provides a number of applications with speech recognition implementation. Google's voice actions applications enable control of a mobile phone using voice, such as calling Business and contacts, sending texts and email, listening to music, browsing the web, and completing common tasks. The feature like sending SMS in Regional Languages is a requirement of present day mobile users with increasing use of chatting messengers like What's App etc. as people from different communities are interested more in sending messages in their own regional languages. This also is particularly useful for sending wishes on special culture related festivals like Ugadi for Telugu People, Gudi Padva for Marathi people etc... In their own regional languages. This is a novel idea to implement a transliteration algorithm for generating regional language text correctly in different Indian and foreign languages like Telugu, Hindi, Malayalam, Tamil, Greek, Chinese etc.. , by just typing a phonetic language with ordinary English-us keyboard. When a space is used, the text so typed is converted into the selected language word in a meaningful manner. This enables the user to simply generate his own language text and send it as a message to his friends in a convenient and a fast manner. The requirement of such a product is implemented in this project with following features:
1. Enter App: This is used for Entering the App. Usually called as Welcome Screen of App.

2. Login using Radio Button password: A secure graphical way of giving password to enter into the application.

3. Login using alphanumeric character password: This is additional password in text format for added security.

4. Select option: This is used to select the option we want to perform like Sending SMS, setting/changing radio button password/ alphanumeric Password

5. Web View Based Transliteration and Message sending user form: For sending regional language text. 


\section{ARCHITECTURE}

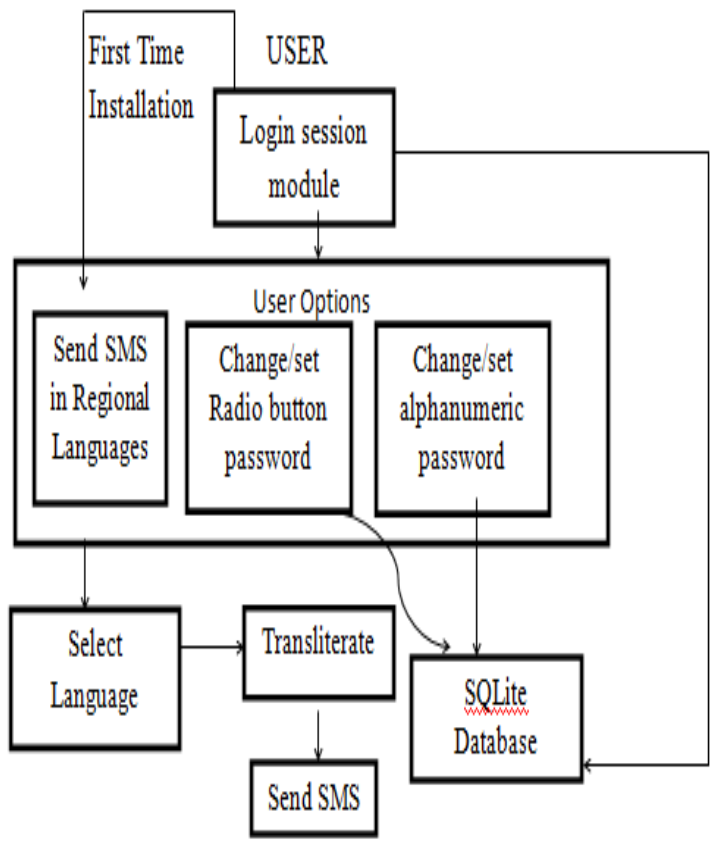

Architecture design is a diagram of a system, in which the principal parts or functions are represented by blocks connected by the lines that show the relationships of blocks. The block diagram is typically used for a higher level, less detailed description aimed more at understanding the overall concepts and less at understanding the details of implementation.

\section{WORKDONE}

\subsection{Password Module}

There are two types of passwords that can be chosen by the user for securing the application. This is used for authentication of the user from the database once the user sets the password. The two types of passwords are:

- Radio Button Password

- Alphanumeric password

\subsection{Transliterating Module}

This module is for transliterating the English phonetics into selected language using transliteration method. This method is loaded in web view.

\subsection{Send SMS Module}

This module is for sending transliterated SMS to the number that user wants to. This app gets permission from android device manager to read contacts in mobile to send SMS

\subsection{Change Password Module}

This module helps in changing the password for the application by the user. Before changing the password user needs to confirm old password if any, then it is verified in database and updated password is replaced in database. This includes change to any of the two passwords.

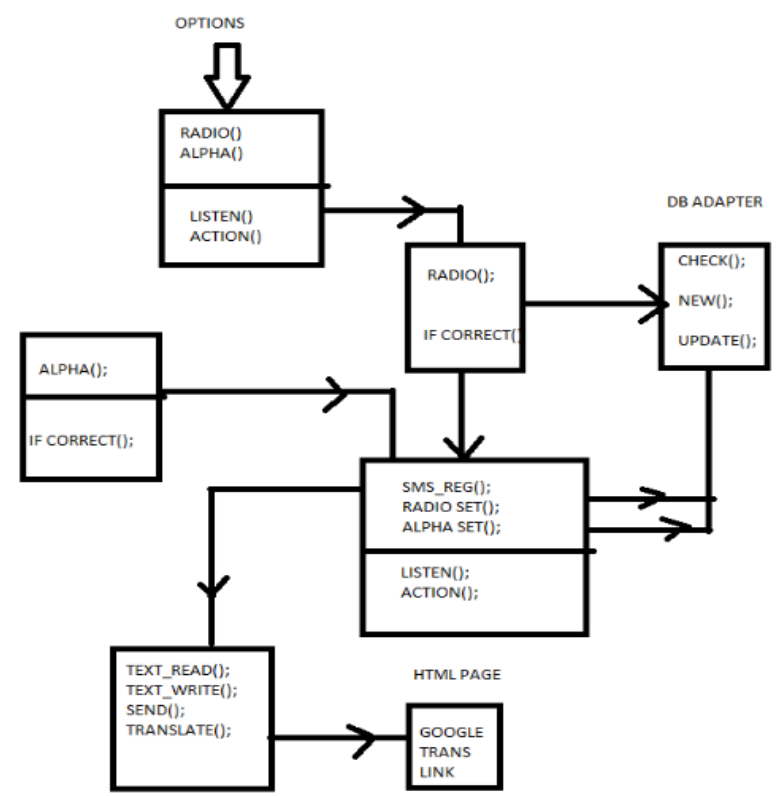

\section{RESULTS}

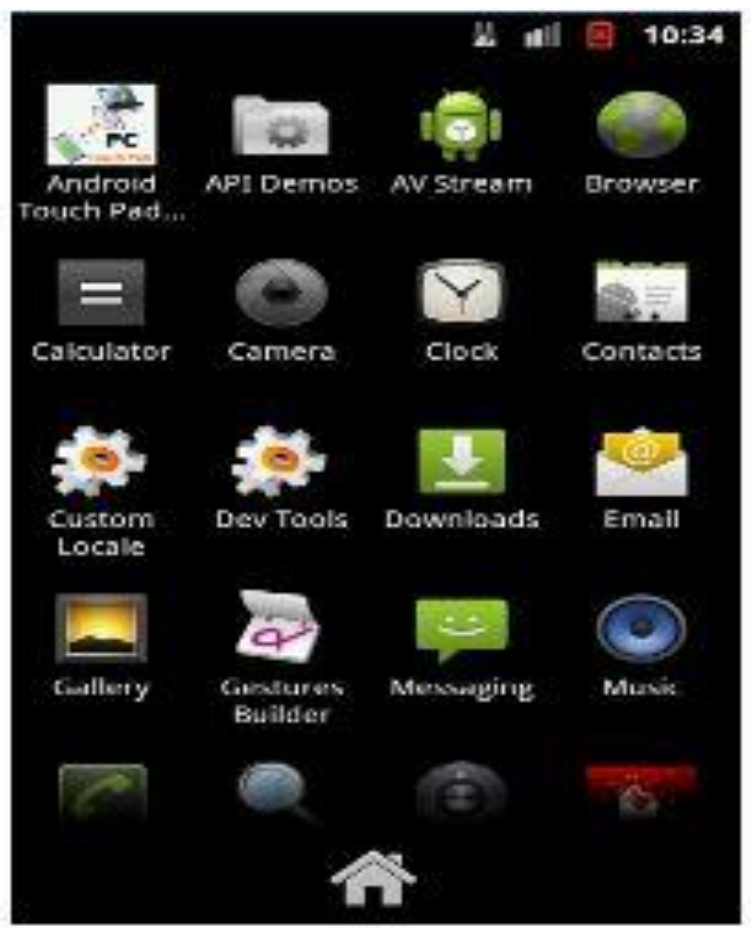

Phone Main Menu 


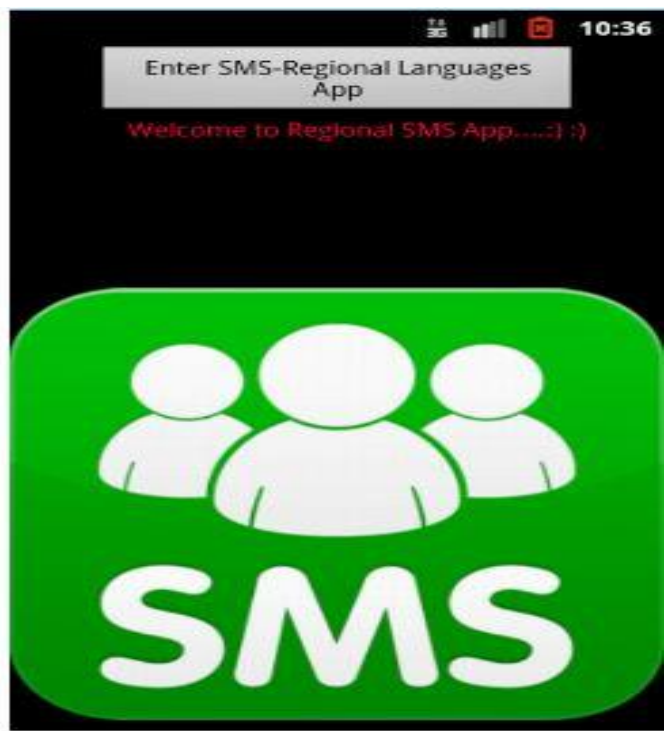

Welcome Screen of Sending SMS in Regional Languages App

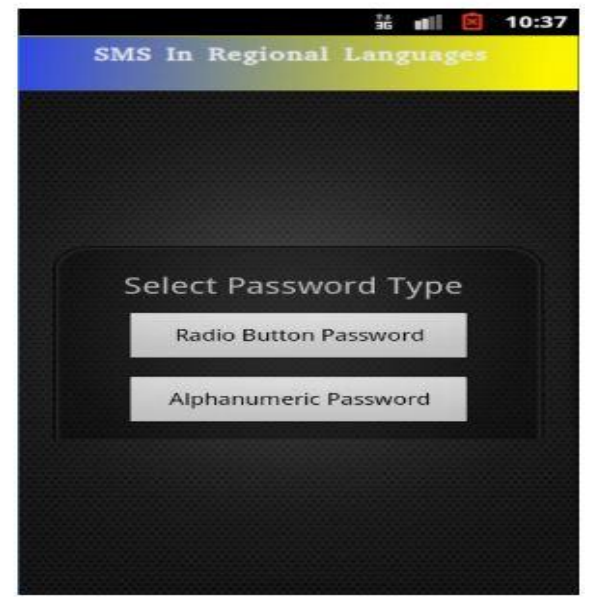

Login Page of App

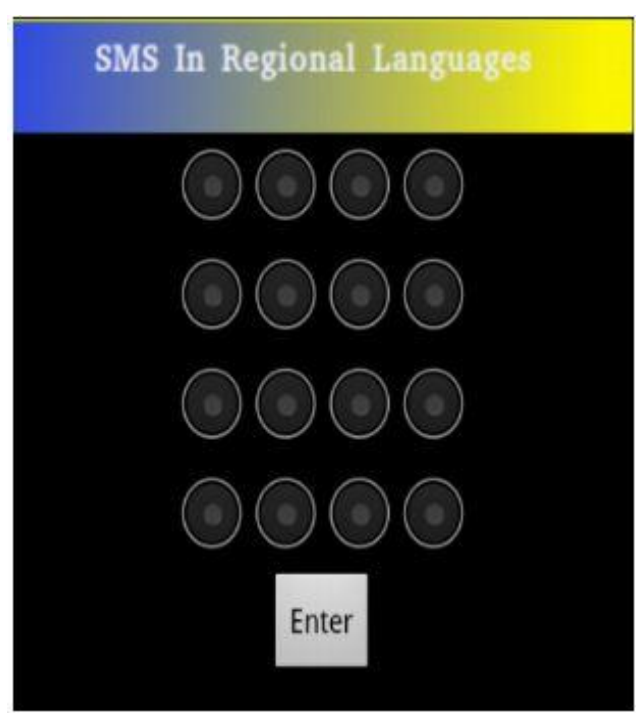

Login Using Radio Button Password

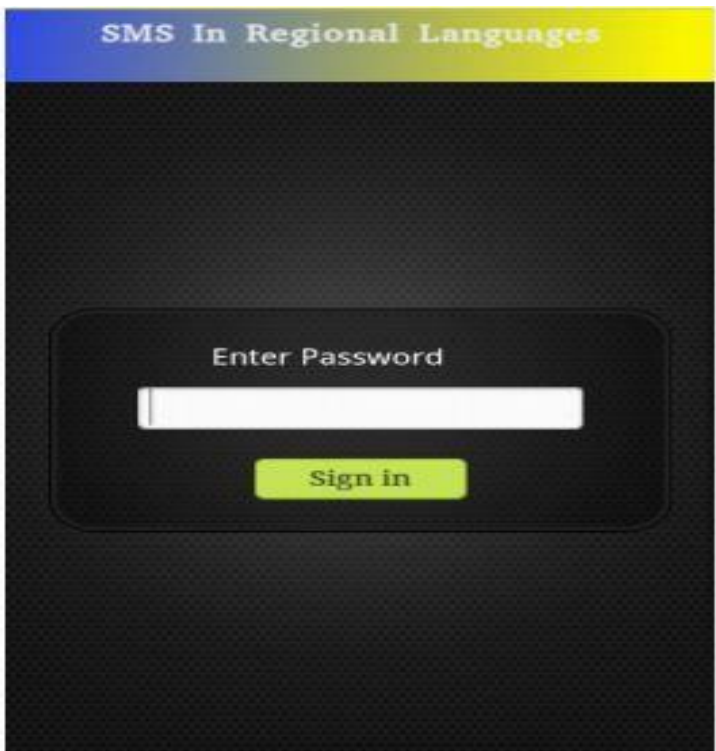

Login Using Alphanumeric Password

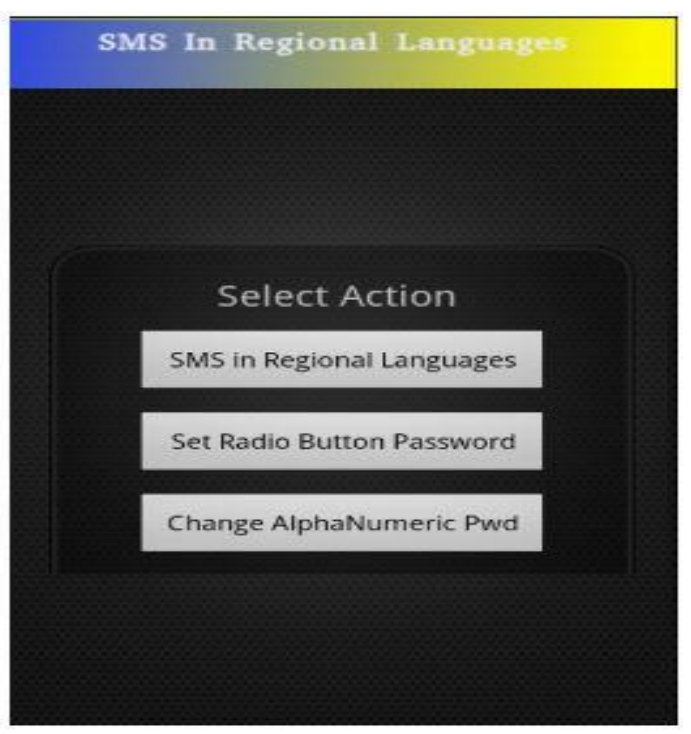

Select Action

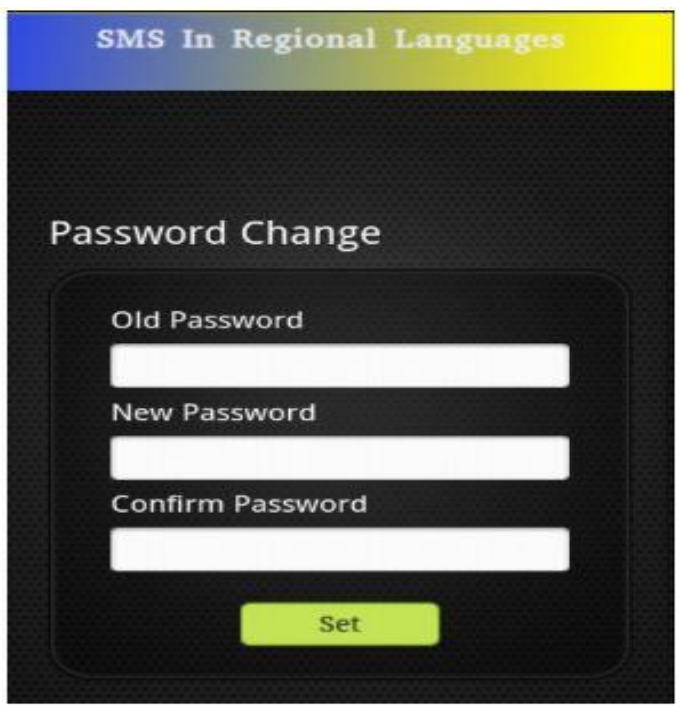

Change Password for App 


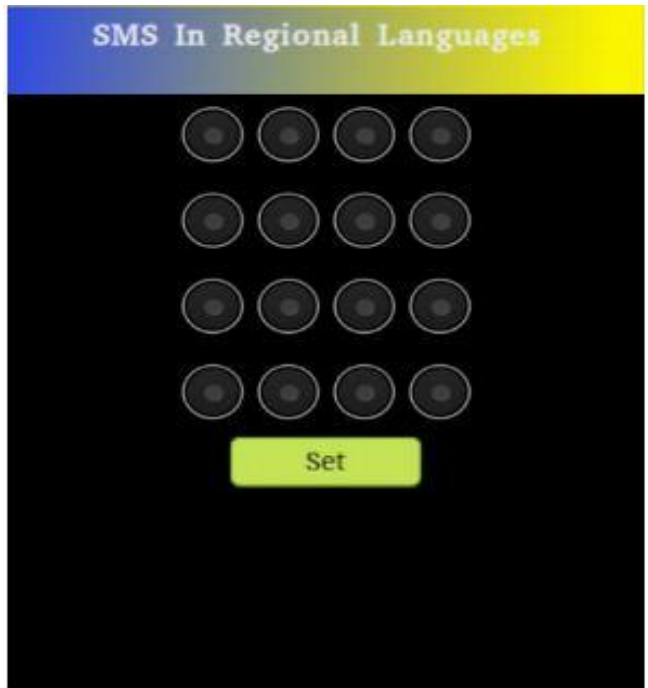

Set Radio Button Password for App

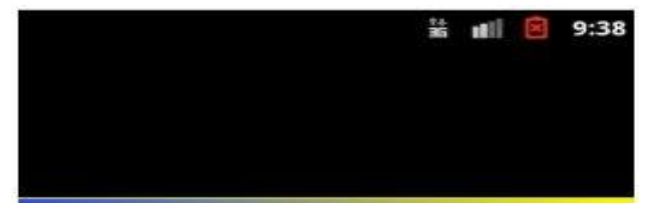

SMS In Regional Languas:

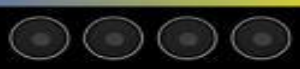

(0)0

0000

0@0

Enter

Invalid Graphical Password!!!!!!

Invalid Graphical Password!ll!

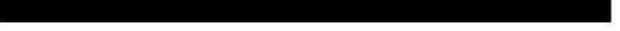

Toast Message of Invalid Graphical password

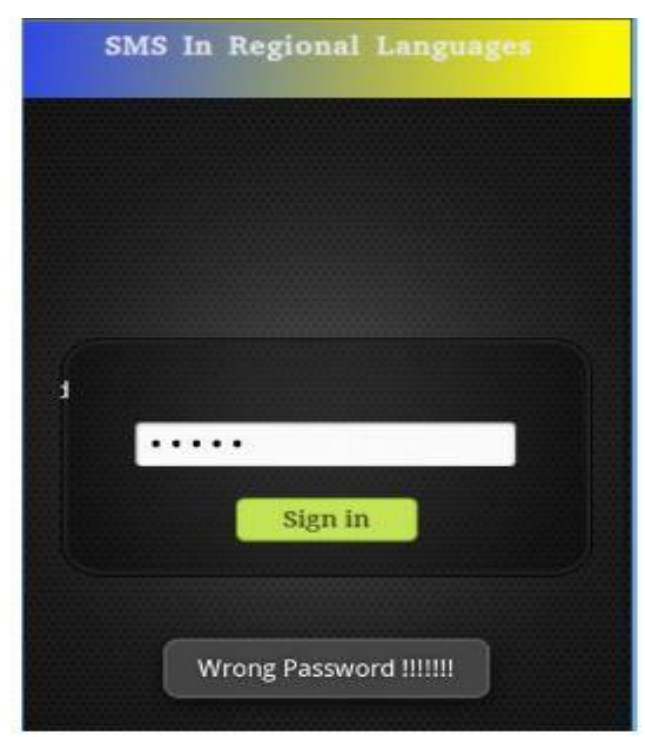

Toast Message of Wrong Password

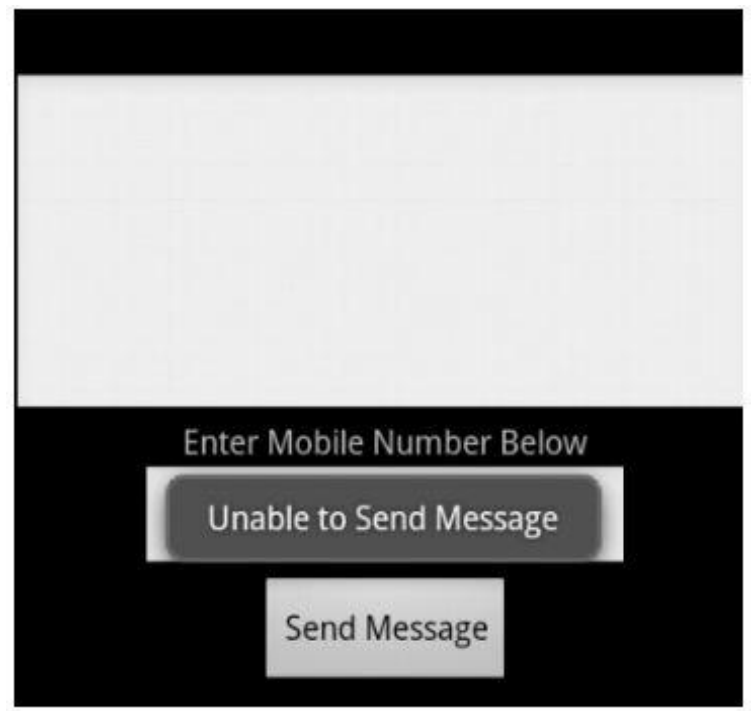

Toast Message for Unable to send Message
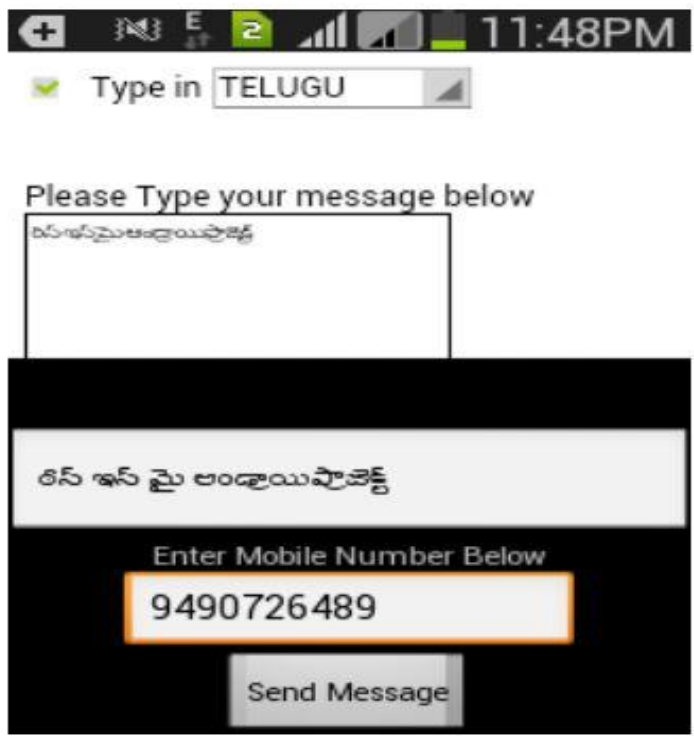

Sending SMS in Regional Languages App

\section{CONCLUSION}

With the development of software and hardware capabilities of mobile devices, there is an increased need for device specific content, what resulted in market changes. Sending SMS in regional languages is of particular interest due to the direct support of communication between human and computers Using Regional SMS application, which works over internet, allows much faster message transliteration. Another advantage is the much larger databases that are used. Many believe that this mode is a big step for SMS technology. The accuracy of system has significantly increased and become more accessible to everyone. We have been given a possibility to manage mobile devices without installing complex software for sending SMS results in memory savings. The project is a development of an Android based App that really converts phonetic text typed by the user into the language selected by him in a dropdown provided for the user to select his particular language of interest. The objective for the feature is development of models and databases for multiple languages which could 
create a foundation for everyday use of this technology worldwide. The main goal of application is to allow sending of text messages in regional languages. Testing has shown simplicity of use and high accuracy of message transliterating. Results of application give user an option to select the most accurate result. Further work is planned to implement the model of speech recognition for different language. The application of transliteration algorithm is the most convenient way of converting any phonetic text typed on English keyboard into a particular regional language. This feature is being incorporated in this App for sending SMS in Regional Languages. The user can choose 18 different languages other than English for typing his text and then he can send it as SMS to his friends.

\section{FUTURE SCOPE}

Experiencing the new modern paradigm shifts in technology will require humans to become one with the technologies they create and the ability to interface in real-time. Perhaps the greatest step towards this goal is transliteration technology, where we can transliterate English phonetics into regional languages. It is the time to start discussing some of the potential future applications or killer apps that are now possible or will be shortly, along with issues of funding the research forward. We must consider how other complimentary artificial intelligence advances will hyper advance transliteration performance. This project helps the user to send messages in 18 different languages. This can be further extended considering many other languages. This project also provides security to the app in two ways, radio button password and alphanumeric password. In future the security can be extended to different levels such as finger scan, voice recognition. In future this App could be integrated to other Apps like What's App for example for providing multi-language feature for the App.

\section{REFRENCES}

[1] “Android Developers", http://developer.android.com

[2] Android 2.2 SDK, http://developer.android.com/reference/packages.htm 1

[3] Eclipse 4.2.1, http://www.eclipse.org/galileo/.

[4] Herbert schildt, "java complete reference", $7^{\text {th }}$ edition, publish: Tata McGraw-Hill Education (2006)

[5] http://androidcommunity.com

[6] http://openhandsetalliance.com

[7] http://androidappsdocs.appspot.com/sdk/index.html

[8] http://dlssl.google.com/android/eclipse/ 